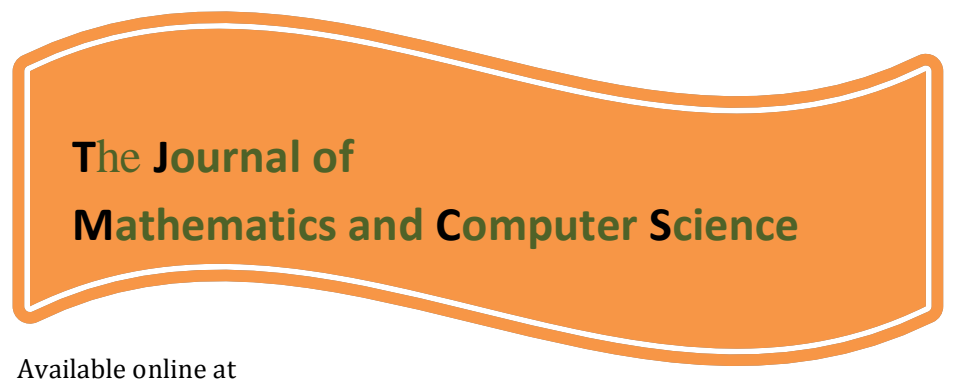

http://www.TIMCS.com

The Journal of Mathematics and Computer Science Vol .3 No.2 (2011) 135 - 144

\title{
A new analytical approach to solve exponential stretching sheet problem in fluid mechanics by variational iterative Padé method
}

\author{
Majid Khan ${ }^{1},{ }^{a}$, Muhammad Asif Gondal ${ }^{a}$, and Sunil Kumar ${ }^{b}$ \\ ${ }^{a}$ Department of Science and Humanities, National University of Computer and \\ Emerging Science Islamabad, Pakistan \\ ${ }^{b}$ Department of Applied Mathematics, Institute of Technology Banaras \\ Hindu University, India
}

Received: April 2011, Revised: June 2011

Online Publication: July 2011

\begin{abstract}
:
In this article, we present a reliable combination of variational iterative method and Padé approximants to investigate two dimensional exponential stretching sheet problem. The proposed method is called variational iterative Pade' method (VIPM). The method is capable of reducing the size of calculation and easily overcomes the difficulty of perturbation methods or Adomian polynomials. The results reveal that the VIPM is very effective and is easy to apply.
\end{abstract}

Keywords: Variational iterative method, Pade' approximation, Exponential stretching sheet, Similarity transforms, Series solution.

Corresponding author:

Fax:. +92 0514100619 .

Tel:. +92 03345404638.

E-mail Address: mk.cfd1@gmail.com. 


\section{Introduction}

Due to numerous industrial processes the boundary layer concept for flow of an incompressible fluid over a exponential stretching sheet is quite popular among the researchers recently. Such processes include aerodynamic extrusion of plastic sheets, hot rolling, gluing of labels on hot bodies, fiber-glass, paper production and applications in polymer industries. A broad range of analytical and numerical methods have been used in the analysis of these scientific models. Mathematical modeling of many physical systems leads to nonlinear ordinary or partial differential equations in various fields of physics and engineering. An effective method is required to analyze the mathematical model which provides solutions conforming to physical reality. Common analytic procedures linearize the system or assume that nonlinearities are relatively insignificant. Such assumptions, sometimes strongly, affect the solution with respect to the real physics of the phenomenon. Thus seeking exact solutions of nonlinear ordinary or partial differential equation is of great importance. Various powerful mathematical techniques such as Adomian decomposition method [1-5], homotopy perturbation method [6-10], and Laplace decomposition method [11-15], have been proposed for obtaining exact and approximate analytic solutions.

The variational iteration method (VIM) was first proposed by He [16-20], systematically illustrated in 1999 and used to give approximate solutions of the problem of seepage flow in porous media with fractional derivatives. The VIM is useful to obtain exact and approximate solutions of linear and nonlinear differential equations.

In this method, general Lagrange multipliers are introduced to construct correction functional for the problems. The multipliers can be identified optimally via the variational theory. There is no need for linearization, discretization and large computational work. It has been used to solve effectively, easily and accurately a large class of nonlinear problems with approximation[21-23]. It has been shown by many authors that this method is more powerful than existing techniques such as the Adomian method[24-25]. The Padé approximants, that often show superior performance over series approximations, provide a successful tool and promising scheme for applications[26].

The objective of this article is to use variational iteration method and Padé approximant to solve exponential stretching sheet equations for the two-dimensional steady, laminar and viscous flow. The paper is organized as follows. In Section 2, the basic concepts of VIM is presented. Section 3 contains basic idea of Pade approximants. Section 4, contains governing equations. The conclusions are given in last section.

\section{Variational iterative method}

To illustrate the basic concept of the variational iteration method (VIM), we consider the following general differential equation:

$$
L u+N u=g(x)
$$

where $L$ is a linear operator, $N$ is a nonlinear operator, and $g(x)$ is the forcing 
term. According to variational iteration method [16-20], we can construct a correction functional as follows:

$$
u_{n+1}=u_{n}+\int_{0}^{x} \lambda(\xi)\left[L u_{n}(\xi)+N u_{n}(\xi)-g(\xi)\right] d \xi \text {, }
$$

where $\lambda$ is a Lagrange multiplier [16-20], which can be identified optimally via variational iteration method. The subscripts $n$ denote the $n$th approximation, $\tilde{u}_{n}$ is considered as a restricted variation, that is, $\delta \tilde{u}_{n}=0$ Relational (2) is called as a correctional functional. The solution of the linear problems can be solved in a single iteration step due to the exact identification of the Lagrange multiplier. The principles of variational iteration method and its applicability for various kinds of differential equations are given in [16-20] . In this method, it is required first to determine the Lagrange multiplier $\lambda$ optimally. The successive approximation $\quad u_{n+1} \quad, n \geq 0$ of the solution $u$ will be readily obtained upon using the determined Lagrange multiplier and any

selective function. Consequently, the solution is given by

$$
u=\lim _{n \rightarrow \infty} u_{n} .
$$

\section{Padé approximants}

A Padé approximant is the ratio of two polynomials constructed from the coefficients of the Taylor series expansion of a function $u(x)$. The $[L / M]$ Padé approximants to a function $u(x)$ are given by (Baker, 1975, [26])

$$
\left[\frac{L}{M}\right]=\frac{P_{L}(x)}{Q_{M}(x)},
$$

where $P_{L}(x)$ is a polynomial of degree at most $L$ and $Q_{M}(x)$ is a degree of at most $M$. The power series in terms of $x$ is given below

$$
\begin{aligned}
& u(x)=\sum_{i=0}^{\infty} a_{i} x^{i}, \\
& u(x)=\frac{P_{L}(x)}{Q_{M}(x)}+O\left(x^{L+M+1}\right) .
\end{aligned}
$$

Determine the coefficients of $P_{L}(x)$ and $Q_{M}(x)$ by Eq. (6). We can multiply the numerator and denominator by a constant and leave $[L / M]$ unchanged, we imposed the normalization condition as follows 


$$
Q_{M}(x)=1
$$

Expanding polynomials $P_{L}(x)$ and $Q_{M}(x)$ in power series in terms of $x$ of order $L$ and $M$ which is given below :

$$
\begin{gathered}
P_{L}(x)=p_{0}+p_{1} x+p_{2} x^{2}+\ldots+p_{L} x^{L}, \\
Q_{M}(x)=1+q_{1} x+q_{2} x^{2}+\ldots+q_{M} x^{M} .
\end{gathered}
$$

Using Eq. (8) in Eq. (6), we can write Eq. (6) in the notation of formal power series as

$$
\sum_{i=0}^{\infty} a_{i} x^{i}=\frac{p_{0}+p_{1} x+p_{2} x^{2}+\ldots+p_{L} x^{L}}{1+q_{1} x+q_{2} x^{2}+\ldots+q_{M} x^{M}}+O\left(x^{L+M+1}\right) .
$$

By cross-multiplication of Eq. (9) , we get

$$
\left(p_{0}+p_{1} x+\ldots+p_{L} x^{L}\right)\left(a_{0}+a_{1} x+a_{2} x^{2}+a_{3} x^{3}+\ldots\right)=1+q_{1} x+\ldots+q_{M} x^{M}+O\left(x^{L+M+1}\right) .
$$

From Eq. (10), we obtain the set of linear equations

$$
\left\{\begin{array}{c}
a_{0}=p_{0}, \\
a_{1}+a_{0} q_{1}=p_{1}, \\
a_{2}+a_{1} q_{1}+a_{0} q_{2}=p_{2}, \\
\vdots \\
a_{L}+a_{L-1} q_{1}+a_{0} q_{L}=p_{L},
\end{array}\right.
$$

and

$$
\left\{\begin{array}{c}
a_{L+1}+a_{L} q_{1}+\ldots+a_{L-M+1} q_{M}=0, \\
a_{L+2}+a_{L+1} q_{1}+\ldots+a_{L-M+2} q_{M}=0, \\
\vdots \\
a_{L+M}+a_{L+M-1} q_{1}+a_{L} q_{M}=0 .
\end{array}\right.
$$

From Eq. (12), we can obtain $q_{i}, 1 \leq i \leq M$. Once the values of $q_{1}, q_{2}, \ldots, q_{M}$ are all known Eq. (11), gives an explicit formula for the unknown quantities $p_{1}, p_{2}, \ldots, p_{L}$. We calculate diagonal approximants which are more accurate than non diagonal approximants and can be calculated easily by built-in utilities of Mathematica and Maple.

\section{Mathematical formulation of the problem}

Let us consider flow of an incompressible viscous fluid over exponential stretching sheet at 
$y=0$. Under the assumptions of boundary layer theory the continuity and momentum equations are given by

$$
\begin{gathered}
\frac{\partial u}{\partial x}+\frac{\partial v}{\partial y}=0 \\
u \frac{\partial u}{\partial x}+v \frac{\partial u}{\partial y}=v \frac{\partial^{2} u}{\partial x^{2}}
\end{gathered}
$$

where $u$ and $v$ are the velocity components in the $x$ and $y$ directions respectively, $\quad v \quad$ is the kinematic viscosity which is the ratio of dynamic viscosity to the density of the fluid i.e. $v=\mu / \rho$. The boundary conditions corresponding to the exponential stretching sheet are:

$$
\begin{aligned}
& u=U_{0} e^{\frac{x}{L}}, v=0, \text { at } y=0, \\
& u \rightarrow 0 \text { as } y \rightarrow \infty,
\end{aligned}
$$

where $U_{0}$ is the reference velocity and $L$ is a constant. Upon making use of the following similarity transformations in Eqs. (13) - (16) :

$$
\eta=\sqrt{\frac{U_{0}}{2 v L}} e^{\frac{x}{L}} y, u=U_{0} e^{\frac{x}{L}} f^{\prime}(\eta), v=-\sqrt{\frac{v U_{0}}{2 L}} e^{\frac{x}{L}}\left[f(\eta)+\eta f^{\prime}(\eta)\right] .
$$

The resulting non-linear differential equations with corresponding boundary conditions are of the following form

$$
\begin{gathered}
f^{\prime \prime \prime}-2 f^{\prime 2}+f f^{\prime \prime}=0, \\
f(0)=0, \quad f^{\prime}(0)=1, \quad f^{\prime}(\infty)=0 .
\end{gathered}
$$

In this section, we mainly solve our governing equation (18) with boundary condition given in Eq. (19), by He's variational iterative method. The initial guess for $f$ is given below

$$
f_{0}(\eta)=\eta+\frac{\alpha \eta^{2}}{2}
$$

where $f^{\prime \prime}(0)=\alpha<0$. To solve Eq. (18)-(19), with the help of variational iterative method, we construct a correctional functional which is given below

$$
f_{n+1}(\eta)=f_{n}(\eta)+\int_{0}^{\eta} \lambda(\xi)\left[\frac{\partial^{3} f_{n}(\xi)}{\partial \xi^{3}}-2\left(\tilde{f}_{n}^{\prime}(\xi)\right)^{2}+\tilde{f}_{n}(\xi) \tilde{f}_{n}^{\prime \prime}(\xi)\right] d \xi
$$


where $\lambda$ is a Lagrangian multiplier which can be determine optimally and $\tilde{f}_{n}(\xi)$ is considered as a restricted variation i-e., $\quad \tilde{\delta}_{n}(\xi)=0$. To find the optimal values of $\lambda$ we have

$$
\delta f_{n+1}(\eta)=\delta f_{n}(\eta)+\delta \int_{0}^{\eta} \lambda(\xi)\left[\frac{\partial^{3} f_{n}(\xi)}{\partial \xi^{3}}-2\left(\tilde{f}_{n}^{\prime}(\xi)\right)^{2}+\tilde{f}_{n}(\xi) \tilde{f}_{n}^{\prime \prime}(\xi)\right] d \xi
$$

or

$$
\delta f_{n+1}(\eta)=\delta f_{n}(\eta)+\delta \int_{0}^{\eta} \lambda(\xi)\left(\frac{\partial^{3} f(\xi)}{\partial \xi^{3}}\right) d \xi .
$$

Following [16], the stationary conditions are given below

$$
\left\{\begin{array}{c}
\lambda^{\prime \prime \prime}(\xi)=0, \\
\left.\lambda^{\prime \prime}(\xi)\right|_{\xi=\eta}+1=0, \\
\left.\lambda^{\prime}(\xi)\right|_{\xi=\eta}=0 \\
\left.\lambda(\xi)\right|_{\xi=\eta}=0
\end{array}\right.
$$

On solving above Eq. (24), we get

$$
\lambda=-\frac{1}{2}(\xi-\eta)^{2} .
$$

Substituting this values of Lagrange multiplier in functional (21), we get an iterative formula

$$
f_{n+1}(\eta)=f_{n}(\eta)-\frac{1}{2} \int_{0}^{\eta}(\xi-\eta)^{2}\left[\frac{\partial^{3} f_{n}(\xi)}{\partial \xi^{3}}-2\left(\tilde{f}_{n}^{\prime}(\xi)\right)^{2}+\tilde{f}_{n}(\xi) \tilde{f}_{n}^{\prime \prime}(\xi)\right] d \xi, \quad n \geq 0
$$

Now the components of the series solution are

$$
\begin{aligned}
& f_{0}(\eta)=\eta+\frac{\alpha \eta^{2}}{2}, \\
& f_{1}(\eta)=\eta+\frac{\alpha \eta^{2}}{2}+\frac{\eta^{3}}{3}+\frac{\alpha \eta^{4}}{8}+\frac{\alpha^{2} \eta^{5}}{40},
\end{aligned}
$$




$$
\begin{aligned}
f_{2}(\eta)= & \eta+\frac{\alpha \eta^{2}}{2}+\frac{\eta^{3}}{3}+\frac{\alpha \eta^{4}}{8}+\frac{\eta^{5}}{30}+\frac{\alpha^{2} \eta^{5}}{40}+\frac{19 \alpha \eta^{6}}{720} \\
& +\frac{2 \eta^{7}}{315}+\frac{3 \alpha^{2} \eta^{7}}{560}+\frac{5 \alpha \eta^{8}}{1344}+\frac{3 \alpha^{3} \eta^{8}}{4480}+\frac{143 \alpha^{2} \eta^{9}}{120960} \\
& +\frac{\alpha^{3} \eta^{10}}{4800}+\frac{\alpha^{4} \eta^{11}}{52800}
\end{aligned}
$$

The series solution is given by

$$
\begin{gathered}
f(\eta)=\lim _{n \rightarrow \infty} f_{n}, \\
f(\eta)=\eta+\frac{\alpha \eta^{2}}{2}+\frac{\eta^{3}}{3}+\frac{\alpha \eta^{4}}{8}+\frac{\eta^{5}}{30}+\frac{\alpha^{2} \eta^{5}}{40}+\frac{19 \alpha \eta^{6}}{720}+\frac{2 \eta^{7}}{315} \\
+\frac{3 \alpha^{2} \eta^{7}}{560}+\frac{5 \alpha \eta^{8}}{1344}+\frac{3 \alpha^{3} \eta^{8}}{4480}+\frac{143 \alpha^{2} \eta^{9}}{120960}+\frac{\alpha^{3} \eta^{10}}{4800}+\frac{\alpha^{4} \eta^{11}}{52800}+\ldots
\end{gathered}
$$

Our aim in this section is mainly concerned with the mathematical behavior of the solution $f(\eta)$ in order to determine the value of free parameter $\alpha=f^{\prime \prime}(0)$. It was formally shown by that this goal can easily be achieved by forming the Padé approximants [26] which have the advantage of manipulating the polynomial approximation into a rational function to obtain the more information about $f(\eta)$. It is well known fact that Padé approximants will converges on the entire real axis if $f(\eta)$ is free of singularities on the entire real axis. More importantly, the diagonal approximants are most accurate approximants, therefore we will construct only diagonal approximants. Using the boundary condition $f^{\prime}(\infty)=0$, the diagonal approximants $[M / M]$ vanish if the coefficients of $\eta$ with the highest power in the numerator vanishes. Choosing the coefficients of the highest power of $\eta$ equal to zero, we get a polynomial equations in $\alpha$ which can be solved very easily by using the built in utilities in the most manipulation languages.

Table. 1: Numerical values of free parameter $\alpha=f^{\prime \prime}(0)$.

\begin{tabular}{|lc|}
\hline Padé approximants & Present Method (VIPM) \\
\hline$[21 / 21]$ & -1.28138 \\
{$[22 / 22]$} & -1.28075 \\
{$[23 / 23]$} & -1.27982 \\
{$[24 / 24]$} & -1.28028 \\
{$[25 / 25]$} & -1.28217 \\
{$[26 / 26]$} & -1.28184 \\
{$[27 / 27]$} & -1.28181 \\
{$[28 / 28]$} & -1.28181 \\
{$[29 / 29]$} & -1.28181 \\
{$[30 / 30]$} & -1.28181 \\
& \\
\hline
\end{tabular}




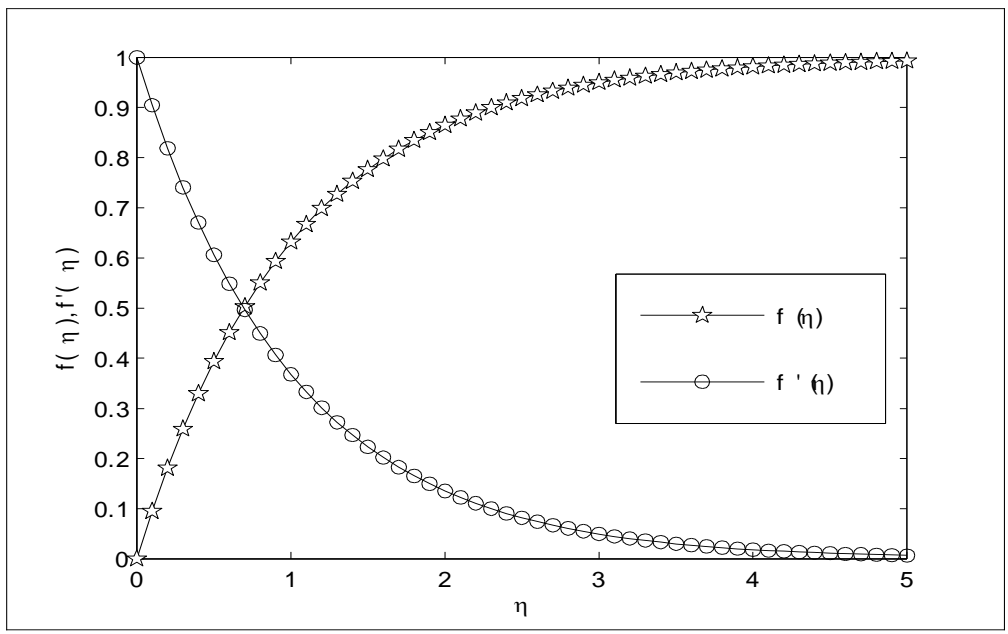

Fig. 1: Solution and velocity curves by VIPM: Line with stars indicates $10^{\text {th }}$-order solution curve and line with circles shows [10/10] Padé velocity curve.

Table 1 and Fig. 1, clearly elucidates that present solution method namely VIPM shows fast convergent results. This analysis shows that VIPM suits for exponential flow problems and with the use of Padé approximants we accelerate convergence of our obtained series.

\section{Concluding Remarks}

The main aim of this work is to provide the series solution of the boundary layer equation of two-dimensional flow over a exponential stretching sheet by using variational iterative Padé method (VIPM). The new proposed variational iterative Padé method (VIPM) is a powerful tool to search for solutions of various nonlinear problems. The method overcomes the difficulty in other methods because it is efficient. We derived fast convergent results by combining the series, obtained by the variational iterative method, with the diagonal Padé approximants. The convergence of VIPM is also shown in Table 1 . The analysis given here shows confidence on VIPM.

\section{References}

1. G. Adomian, The decomposition method, Boston: Kluwer Acad. Publ. 1994 .

2. M. M. Hosseini, Adomian decomposition method with Chebyshev polynomials, Appl. Math. Comput. 175 (2006) 1685-1693.

3. M. M. Hosseini, Numerical solution of ordinary differential equations with impulse solution, Appl. Math. Comput. 163 (2005) 373-381.

4. M. M. Hosseini, Adomian decomposition method for solution of differential-algebraic 
equations, J. Comput. Appl. Math. 197 (2006) 373-381.

5. M. M. Hosseini, Adomian decomposition method for solution of nonlinear differential algebraic equations, Appl. Math. Comput. 181 (2006) 1737-1744.

6. J. H. He, Homotopy perturbation technique, Comput. Meth. Appl. Mech. Eng. 178 (1999) $257-262$.

7. J. H. He, A coupling method of a homotopy technique and a perturbation technique for non-linear problems, Int. J. Nonlin. Mech. 35 (2000) 37-43.

8. J. H. He, Approximate Solution of Nonlinear Differential Equations With Convolution Product Nonlinearities, Comput. Meth. Appl. Mech. Eng. 167 (1998) 69-73.

9. H. Jafari, M. Zabihi and M. Saidy, Application of Homotopy-Perturbation Method for Solving Gas Dynamics Equation, Appl. Math. Sci. 2 (2008) 2393-2396.

10. D. D. Ganji, The applications of He's homotopy perturbation method to nonlinear equation arising in heat transfer, Phy. Lett. A. 335 (2006) 337-3341.

11. Y. Khan, An Effective Modification of the Laplace Decomposition Method for Nonlinear Equations, Int. J. Nonlinear Sci. Numer. Simul. 10 (2009) 1373-1376.

12. M. Hussain, M. Khan, Modified Laplace Decomposition Method, Appl. Math. Sci. 4 (2010) 1769-1783.

13. M. Khan, M. Hussain, Application of Laplace decomposition method on semi infinite domain. Numer. Algor. 56 (2011) 211-218.

14. M. Khan, M. A. Gondal, New modified Laplace decomposition algorithm for Blasius flow equation, J. Adv. Res. Sci. Comput. 2 (2010) 35-43.

15. M. Khan, M. A. Gondal, A new analytical solution of foam drainage equation by Laplace decomposition method, J. Adv. Res. Diff. Eqs. 2 (2010) 53-64.

16. J. H. He, Approximate Analytical Solution for Seepage Flow with Fractional Derivatives in Porous Media, Comput. Meth. Appl. Mech. Eng. 167 (1998) 57-68.

17. J. H. He, X.-H.Wu, Variational iteration method: new development and applications, Comput. Math. Appl. 54 (2007) 881-894.

18. J. H. He, The variational iteration method for eighth-order initial-boundary value problems, Phy. Scri. 76 (2007) 680-682.

19. J. H. He, Variational iteration method-a kind of non-linear analytical technique: some examples, Int. J. Nonlin. Mech. 34 (1999) 699-708.

20. M. Hussain, M. Khan, A Variational Iterative Method for Solving the Linear and Nonlinear Klein-Gordon Equations, Appl. Math. Sci. 4 (2010) 1931-1940.

21. H. Jafari, A. Yazdani, Javad Vahidi, D. D. Ganji, Application of He's Variational Iteration Method for Solving Seventh Order Sawada-Kotera Equations, Appl. Math. Sci. 2 (2008) $471-477$.

22. H. Jafari, H. Tajadodi, He's Variational Iteration Method for Solving Fractional Riccati Differential Equation, Int. J. Diff. Eqs. (2010) , doi:10.1155/2010/764738.

23. H. Jafari, M. Zabihi, E. Salehpoor, Application of variational iteration method for modified Camassa-Holm and Degasperis-Procesi equations, Numer. Meth. Part. Diff. Eqs. 26 (2010) 1033-1039. 
Majid Khan, Muhammad Asif Gondal and Sunil Kumar / TJMCS Vol .3 №.2 (2011) 135 - 144

24. H. Jafari, A. Alipoor, A new method for calculating general Lagrange multiplier in the variational iteration method, Numer. Meth. Part. Diff. Eqs. (2010), doi: 10.1002/num.20567.

25. H. Jafari, A. Golbabaib, E. Salehpoorc, Kh. Sayehvandb, Application of Variational Iteration Method for Stefan Problem, Appl. Math. Sci. 2 (2008) 3001-3004.

26. G. A. Baker. Essentials of Padé Approximants, Academic Press, London, 1975. 